Research Article

\title{
Direction of Arrival Based on the Multioutput Least Squares Support Vector Regression Model
}

\author{
Kai Huang $\mathbb{D}^{1,2}$ Ming-Yi You ${ }^{1},{ }^{1,2}$ Yun-Xia Ye, ${ }^{1,2}$ Bin Jiang, ${ }^{1,2}$ and An-Nan Lu ${ }^{1,2}$ \\ ${ }^{1}$ Science and Technology on Communication Information Security Control Laboratory, Jiaxing 314033, Zhejiang, China \\ ${ }^{2}$ No. 36 Research Institute of CETC, Jiaxing 314033, Zhejiang, China \\ Correspondence should be addressed to Ming-Yi You; youmingyi@126.com
}

Received 14 July 2020; Revised 15 September 2020; Accepted 19 September 2020; Published 30 September 2020

Academic Editor: Liangtian Wan

Copyright (c) 2020 Kai Huang et al. This is an open access article distributed under the Creative Commons Attribution License, which permits unrestricted use, distribution, and reproduction in any medium, provided the original work is properly cited.

The interferometer is a widely used direction-finding system with high precision. When there are comprehensive disturbances in the direction-finding system, some scholars have proposed corresponding correction algorithms, but most of them require hypothesis based on the geometric position of the array. The method of using machine learning that has attracted much attention recently is data driven, which can be independent of these assumptions. We propose a direction-finding method for the interferometer by using multioutput least squares support vector regression (MLSSVR) model. The application of this method includes the following: the construction of MLSSVR model training data, training and construction of the MLSSVR model, and the estimation of direction of arrival. Finally, the method is verified through numerical simulation. When there are comprehensive deviations in the system, the direction-finding accuracy can be effectively improved.

\section{Introduction}

Direction of arrival (DOA) estimation is a widely studied problem in various fields, including wireless communications [1], radar detection [2-4], target localization, and tracking $[5,6]$. Various methods have been proposed to estimate DOA of emitters, such as interferometer $[7,8]$ and array processing [9-11].

The interferometer estimates the DOA based on the phase difference of different direction-finding baselines. The accuracy of interferometer is sensitive to the phase difference of baselines. In engineering applications, there may be various deviations, such as phase inconsistency between channels, mutual coupling between the antennas, and antenna location deviations. In order to achieve optimal direction-finding performance, the methods including correlation-coefficient [12], weighted least squares [13], and parameter estimation [14] are always used. To facilitate method implementation, simplified models are established to describe the effects of various deviations, and autocalibration processes are proposed to improve DOA estimation precision [15-21]. Most of the simplifications on array deviations are made from mathematical perspectives approximately with various additional assumptions, such as uniform linearity or circularity array geometries [15-17], constrained antennas location deviations within a particular line or plane $[18,19]$, and intersensor independence of gain and phase errors [20, 21].

However, the effect of comprehensive disturbances, which probably exist in practical systems, is much more difficult to be modeled precisely and calibrated automatically. The multiple deviations have a great influence on the amplitude and phase of each receiving channel, which greatly affects the performance of the interferometer direction-finding system. The commonly used method to reduce the effect of multiple deviations is the external field calibration method; i.e., for the different directions of incoming signal with a large signal-to-noise ratio, the measured values of the phase difference from baselines are directly recorded and saved together with the known calibration directions [22]. In the application of the directionfinding system, the DOA is calculated by least squares between the measured value of each phase difference with the saved values from the external field. The external field 
calibration method is simple in principle and easy to operate and has been widely used in engineering. Due to the influence of multiple deviations, even if there are different gains of each antenna, the external field calibration method always uses the equal-weight least squares method. The influence of the inconsistency between the antennas on the direction-finding accuracy has not been fully considered, i.e., the external field calibration method fails to maximize the effectiveness of the direction-finding system.

Recently, some scholars have used machine learning to solve the DOA estimation with comprehensive disturbances [23-31]. Machine learning have significant advantages over traditional methods based on array geometries and least square in solving direction-finding problems in complicated scenarios with multiple deviations, such as radial basis function (RBF) [23], least squares support vector classification (LSSVC) [24], support vector regression (SVR) [25-29], and deep neural networks (DNN) $[30,31]$. These methods are data driven and do not rely on preassumptions about array geometries and whether they are calibrated or not. Despite its potential usefulness, the standard formulation of the LS-SVR cannot cope with the multioutput case [32]. Therefore, with the angles around $0^{\circ}$ and $360^{\circ}$, the accuracy has not been effectively improved. The DNN for direction finding requires large samples to perform well, resulting in too long model training time, and if the samples size is insufficient, this method cannot effectively perform. This paper proposes a direction-finding method for the interferometer based on the multioutput least squares support vector regression (MLSSVR) model. The multioutput mode can avoid large errors in the single-output mode around $0^{\circ}$ and $360^{\circ}$, and it is expected to achieve higher direction-finding accuracy within the angle range. Due to the MLSSVR model, the model training time can be greatly shortened. The application of this method includes the following: (1) the construction of MLSSVR model training data, (2) training and construction of the MLSSVR model, and (3) the estimation of direction of arrival.

The organization of the rest of the paper is as follows. Section 2 formulates the array output model with complicated deviations. Section 3 presents the process of the MLSSVR model for DOA estimation. Section 4 carries out simulations to verify the validity of the method. Section 5 concludes this work.

The main notation used in this paper is listed in Table 1.

\section{Problem Formulation}

Assume that the waveform of signal is $s(t)$ and direction of signal is $\alpha$. Then, the antenna output is

$$
\mathbf{x}(t)=\mathbf{a}(\alpha) s(t)+\mathbf{v}(t)
$$

When the comprehensive disturbances exist in the direction-finding system, such as phase inconsistency between channels, mutual coupling between the antennas, and antenna location deviations, these disturbances cause deviations to $\mathbf{a}(\alpha)$, and the actual antenna output is

$$
\widetilde{\mathbf{x}}(t)=\mathbf{a}(\alpha, e) s(t)+\mathbf{v}(t)
$$

$\mathbf{a}(\alpha, e)$ is the direction vector of array with comprehensive disturbances.

The phase difference between the antennas may be far away from the theoretical value. Obviously, at this time, high-precision direction finding cannot be performed according to the theoretical geometry of the antennas. In this case, the external field calibration method can be used generally. We propose the MLSSVR model to achieve higher-precision direction finding, and this method is data driven and do not rely on preassumptions about array geometries and whether they are calibrated or not.

\section{MLSSVR Model for DOA Estimation}

3.1. Construction Training Data of the MLSSVR Model. For the MLSSVR model, the training data are constructed as a matrix comprising phase differences of baselines and their corresponding vectors related to the direction of incoming signal, which is

$$
\left[\boldsymbol{\varphi}_{t}, \boldsymbol{\eta}_{\text {label }}\right]=\left[\begin{array}{cccc}
\boldsymbol{\varphi}_{t 1} & \boldsymbol{\varphi}_{t 2} & \ldots & \boldsymbol{\varphi}_{t L} \\
\boldsymbol{\eta}_{\text {label } 1} & \boldsymbol{\eta}_{\text {label } 2} & \ldots & \eta_{\text {label } L}
\end{array}\right]^{T},
$$

where $\varphi_{t i}=\left[\begin{array}{lll}\varphi_{t i}^{12} & \ldots & \varphi_{t i}^{(N-1) N}\end{array}\right]^{\mathrm{T}},(i=1, \ldots, L)$ is the phase difference vector of the $i$-th training sample, where $T$ is the transposed symbol, and $\eta_{\text {labeli }}$ is the $i$-th vector which is related to the DOA of the signal. In order to avoid the large direction-finding error around $0^{\circ}$ or $360^{\circ}$ caused by the unreasonable loss function of the single-output SVR model, we use the dual output form, namely,

$$
\eta_{\text {labeli }}=\left[\cos \left(\alpha_{\text {labeli }}\right) \sin \left(\alpha_{\text {labeli }}\right)\right]^{\mathrm{T}} \text {. }
$$

Here, $\alpha_{\text {labeli }}$ is the DOA corresponding to the $i$-th training sample.

It should be pointed out that the MLSSVR model is not acquired through only one experiment, and the acquisition of its training data should be carried out under the condition of similar signal-to-noise ratio in the application scenario. On the other hand, in training data of the MLSSVR model, for a given DOA, it is usually necessary to obtain several samples (generally more than 10 [32]). In addition, the DOA of the signal should cover the angle range in the application as much as possible, namely,

$$
\alpha_{l} \leq \alpha_{\text {labeli }} \leq \alpha_{u}
$$

Here, $\alpha_{u}$ and $\alpha_{l}$ represent the upper and lower limits of the possible direction of arrival, respectively.

If the directions of adjacent known incoming signal in the training samples are not the same, they will differ by a fixed step $\Delta \alpha_{t}$, i.e., $\alpha_{\text {labeli+1 }}=\alpha_{\text {label } i}$ or $\alpha_{\text {labeli+1 }}=\alpha_{\text {label } i}+\Delta \alpha_{t}$. The value should generally be less than the requisite accuracy of direction finding in the application. Between $\alpha_{u}$ and $\alpha_{l}$, the number of sample categories is $M$.

3.2. Train and Build a MLSSVR Model. Given a data set $\left(\mathbf{x}_{i}, \mathbf{y}_{i}\right)_{i=1}^{L}, \mathbf{x}_{i} \in \mathbf{R}^{d}$ and $\mathbf{y}_{i} \in \mathbf{R}^{m}$. The purpose of multioutput 
TABLE 1: Symbol and notation.

\begin{tabular}{lc}
\hline Symbol & Explanation \\
\hline$s(t)$ & Waveform of signal \\
$\mathbf{a}(\alpha)$ & Direction vector of array \\
$\mathbf{x}(t)$ & The theoretical output of antennas \\
$L$ & The number of training samples \\
$\mathbf{R}^{a}$ & $a$-Dimensional real number space \\
$\mathbf{R}_{+}$ & One-dimensional positive real number space \\
$\mathbf{C}$ & Mutual coupling matrix \\
$\varphi_{t i}^{k j}$ & The measured value of phase difference from baselines formed between the $k$-th antenna and the $j$-th antenna in the $i$-th training \\
$\alpha$ & sample \\
$\mathbf{v}(t)$ & Direction of signal \\
$\widetilde{\mathbf{x}}(t)$ & Zero-mean Gaussian noise \\
$N$ & The actually output of antennas \\
$\mathbf{R}^{a \times b}$ & The number of antennas \\
$\mathbf{R}_{+}^{a \times b}$ & $a \times b$-Dimensional real number space \\
$\varphi_{t}$ & The matrix formed by cosine and sine function of DOA in the training data \\
$\eta_{\text {label }}$ & Phase differences matrix in the training data \\
\hline
\end{tabular}

regression is to give a set of input vectors $\mathbf{x} \in \mathbf{R}^{d}$ and predict a set of output vectors $\mathbf{y} \in \mathbf{R}^{m}$. The MLSSVR model solves this problem by finding $\mathbf{W}=\left(\mathbf{w}_{1}, \ldots, \mathbf{w}_{m}\right) \in \mathbf{R}^{n_{h} \times m}$ and $\mathbf{b}=$ $\left(b_{1}, \ldots, b_{m}\right)^{T} \in \mathbf{R}^{m}$ that minimize the following objective function with constraints:

$$
\begin{aligned}
& \min \tau(\mathbf{W}, \Xi)=\frac{1}{2} \operatorname{trace}\left(\mathbf{W}^{T} \mathbf{W}\right)+\gamma \frac{1}{2} \operatorname{trace}\left(\Xi^{T} \Xi\right) \\
& \text { s.t. } \mathbf{Y}=\mathbf{Z}^{T} \mathbf{W}+\operatorname{repmat}\left(\mathbf{b}^{T}, L, 1\right)+\Xi .
\end{aligned}
$$

Here, $\mathbf{Y}=\left[\mathbf{y}_{i}\right] \in \mathbf{R}^{L \times m}, Z=\left(\delta\left(\mathbf{x}_{1}\right), \ldots, \delta\left(\mathbf{x}_{L}\right)\right) \in \mathbf{R}^{n_{h} \times L}$, $\delta(\cdot)$ is the mapping function (kernel function) of $\mathbf{R}^{d} \longrightarrow \mathbf{R}^{n_{h}}$, whose purpose is to transform $\mathbf{x}_{i}$ into a more distinguishable high-dimensional feature space with $n_{h}$ dimensions, $\Xi=\left(\xi_{1}, \ldots, \xi_{m}\right) \in \mathbf{R}_{+}^{L \times m}, \xi_{i}=\left(\xi_{i 1}, \ldots, \xi_{i m}\right)^{T}$ and $\xi_{i j}$ is a slack variable; $\operatorname{trace}(\mathbf{A})=\sum_{i}^{m} A_{i, i}$ where $\mathbf{A}$ is a $m \times m$ matrix, and $\gamma \in \mathbf{R}_{+}$is a regularization parameter.

In order to achieve the solution of (6), the objective function can be constructed as a heuristic Bayesian architecture. Let $\mathbf{w}_{i}=\mathbf{w}_{0}+\mathbf{v}_{i}$, where $\mathbf{w}_{0} \in \mathbf{R}^{n_{h}}$ contains common parameter information and $\mathbf{v}_{i} \in \mathbf{R}^{n_{h}}$ carries the individual information of each sample. To obtain a solution $\mathbf{w}_{0}$, $\mathbf{V}=\left(\mathbf{v}_{1}, \ldots, \mathbf{v}_{m}\right)$, and $\mathbf{b}$, the following objective function with constraints can be constructed:

$$
\begin{aligned}
& \min \tau\left(\mathbf{w}_{0}, \mathbf{V}, \boldsymbol{\Gamma}\right)=\frac{1}{2} \mathbf{w}_{0}^{T} \mathbf{w}_{0}+\frac{1}{2} \frac{\lambda}{m} \operatorname{trace}\left(\mathbf{V}^{T} \mathbf{V}\right)+\gamma \frac{1}{2} \operatorname{trace}\left(\boldsymbol{\Gamma}^{T} \boldsymbol{\Gamma}\right) \\
& \text { s.t. } \quad \mathbf{Y}=\mathbf{Z}^{T} \mathbf{W}+\operatorname{repmat}\left(\mathbf{b}^{T}, L, 1\right)+\boldsymbol{\Gamma} .
\end{aligned}
$$

Among them, $\lambda \in \mathbf{R}_{+}$is another regularization parameter.

Equation (7) can be transformed into Lagrange's equation, and then, the optimal solutions $\mathbf{w}_{0}^{*}, \mathbf{V}^{*}$, and $\mathbf{b}^{*}$ can be achieved using the Karush-Kuhn-Tucker (KKT) optimization conditions. After that, the corresponding decision function is [32]

$$
\begin{aligned}
f(\mathbf{x})= & \delta(\mathbf{x})^{T} \mathbf{W}^{*}+\mathbf{b}^{*^{T}}=\delta(\mathbf{x})^{T} \operatorname{repmat}\left(\mathbf{w}_{0}^{*}, 1, m\right) \\
& +\delta(\mathbf{x})^{T} \mathbf{V}^{*}+\mathbf{b}^{*^{T}} .
\end{aligned}
$$

3.3. DOA Estimate. Given a set of phase difference data $\varphi$, based on equation (8), we have

$$
\begin{aligned}
\boldsymbol{\eta}= & {[\cos (\alpha) \sin (\alpha)]^{T}=\delta(\boldsymbol{\varphi})^{T} \operatorname{repmat}\left(\mathbf{w}_{0}^{*}, 1, m\right) } \\
& +\delta(\boldsymbol{\varphi})^{T} \mathbf{V}^{*}+\mathbf{b}^{*^{T}} .
\end{aligned}
$$

Based on the result of equation (9), the final DOA is estimated as

$$
\alpha=\operatorname{atan} 2(\sin (\alpha), \cos (\alpha))=\operatorname{atan} 2(\boldsymbol{\eta}(2,1), \boldsymbol{\eta}(1,1)) .
$$

Here, atan 2 is the four-quadrant inverse tangent function.

\section{Simulations and Analysis}

This section gives a numerical simulation in a typical scenario to demonstrate the effectiveness of the MLSSVR model applied to direction finding. Consider a 5-element uniform circular array with a radius-to-wavelength ratio of 0.4 . The radial basis kernel function is adopted to solve formula (8): $\kappa(x, z)=\exp \left(-p\|x-z\|^{2}\right) p>0$. After multiple trainings, determine the parameters $\gamma=0.5$ and $\lambda=4$ in formula (7) and the radial basis function parameter $p=1$.

4.1. Phase Inconsistency between Channels. Assume that the RMSE of phase difference under $10 \mathrm{~dB}$ signal-to-noise ratio is $25^{\circ}, 5^{\circ}, 5^{\circ}, 10^{\circ}$, and $15^{\circ}$ in 5 channels, respectively. The training data sets consist of phase difference and cosine and sine functions of each angle from $0^{\circ}$ to $360^{\circ}$ with a step of $1^{\circ}$, and 10 groups of samples are collected per angle, i.e., the number of training data sets $L=3600$. Under the same signal-to-noise ratio, a total of 3600 testing samples of phase 


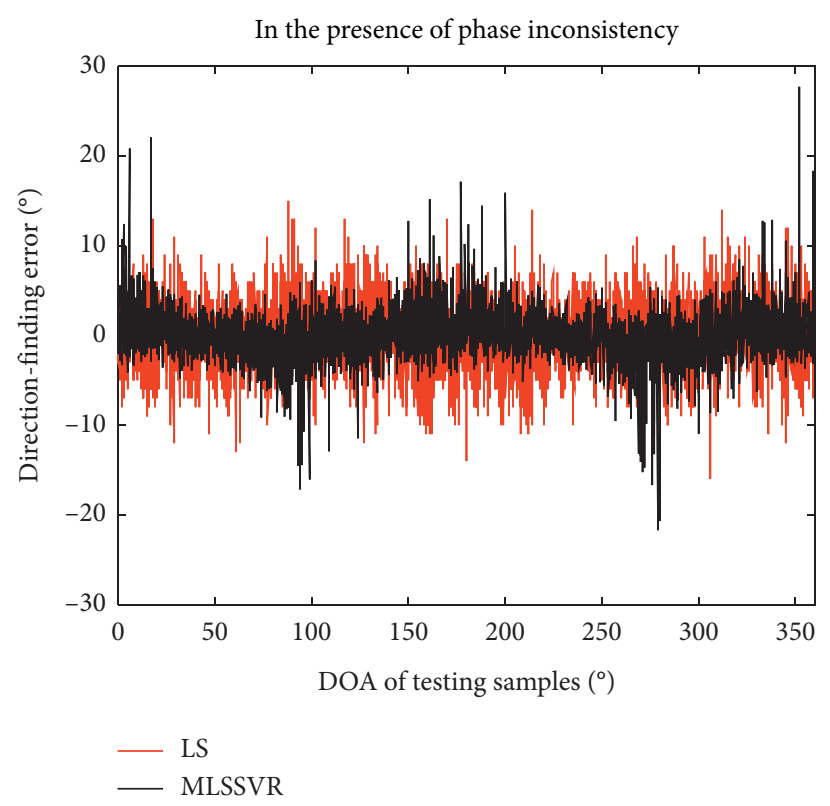

FIGURE 1: Direction-finding error of testing samples with phase inconsistency.

difference are generated from $0^{\circ}$ to $360^{\circ}$. As a comparison, the DOA of testing samples is also calculated by using least squares (LS) with training data sets. Figure 1 shows the direction-finding error of testing samples.
4.2. Mutual Coupling between the Antennas. When there is mutual coupling between antennas, assume the mutual coupling matrix as follows:

$$
\mathbf{C}=\left[\begin{array}{cccccc}
1 & 0.7821+0.2583 j & 0.4576+0.2469 j & 0.4576+0.2469 j & 0.7821+0.2583 j \\
0.7821+0.2583 j & 1 & 0.7821+0.2583 j & 0.4576+0.2469 j & 0.4576+0.2469 j \\
0.4576+0.2469 j & 0.7821+0.2583 j & 1 & 0.7821+0.2583 j & 0.4576+0.2469 j \\
0.4576+0.2469 j & 0.4576+0.2469 j & 0.7821+0.2583 j & 1 & 0.7821+0.2583 j \\
0.7821+0.2583 j & 0.4576+0.2469 j & 0.4576+0.2469 j & 0.7821+0.2583 j & 1
\end{array}\right]
$$

Selecting 10 samples for each angle from $0^{\circ}$ to $360^{\circ}$, and the training data sets consist of phase difference and cosine and sine functions of each angle. Under the same signal-tonoise ratio and mutual coupling, 3600 groups of phase difference from different DOA are generated as the testing samples. Figure 2 shows the direction-finding error of testing samples.

4.3. Both Phase Inconsistency and Mutual Coupling. In the presence of phase inconsistency and mutual coupling simultaneously, 3600 groups of phase difference and cosine and sine functions of DOA are generated as the training data sets for $0^{\circ} \sim 360^{\circ}$ with a step of $1^{\circ}$, and 10 samples are selected for each angle. Under the same signal-to-noise ratio, with the comprehensive disturbances which consist of phase inconsistency and mutual coupling, the testing samples are composed of 3600 groups of phase difference from different DOA. Figure 3 shows the direction-finding error of testing samples.
4.4. Different SNRs. In the presence of phase inconsistency and mutual coupling simultaneously, the training data sets are generated in the same way as mentioned in Section 4.3 under $10 \mathrm{~dB}$ SNR, while testing samples are generated under the $5 \mathrm{~dB}$ SNR. The number of training data sets and testing samples are both 3600 . Figure 4 shows the direction-finding error of testing samples.

Table 2 shows the RMSE of DOA of the testing samples by using LS and MLSSVR, respectively, under different disturbance scenarios. From the results in Table 2, it can be seen that the MLSSVR model can significantly reduce the direction-finding error and obtain high-precision directionfinding results in the full range of $360^{\circ}$.

4.5. Comparison of MLSSVR and Neural Networks. The training data sets and testing samples are generated in the same way as mentioned in Section 4.3. The number of training data sets and the number of testing samples are both 3600 . In additional, both phase inconsistency and 


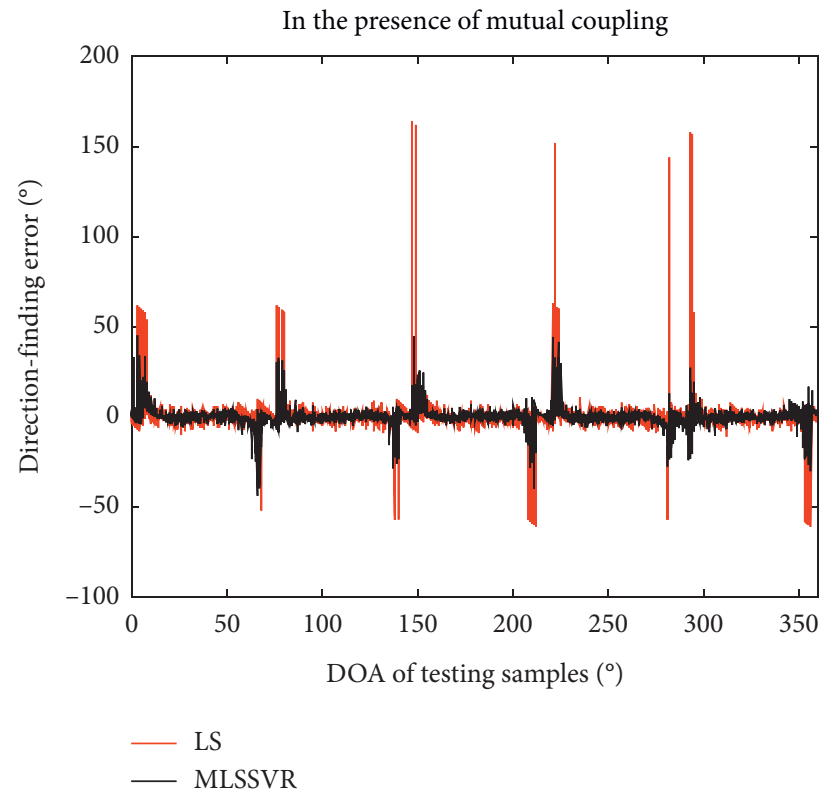

FIGURE 2: Direction-finding error of testing samples with mutual coupling.

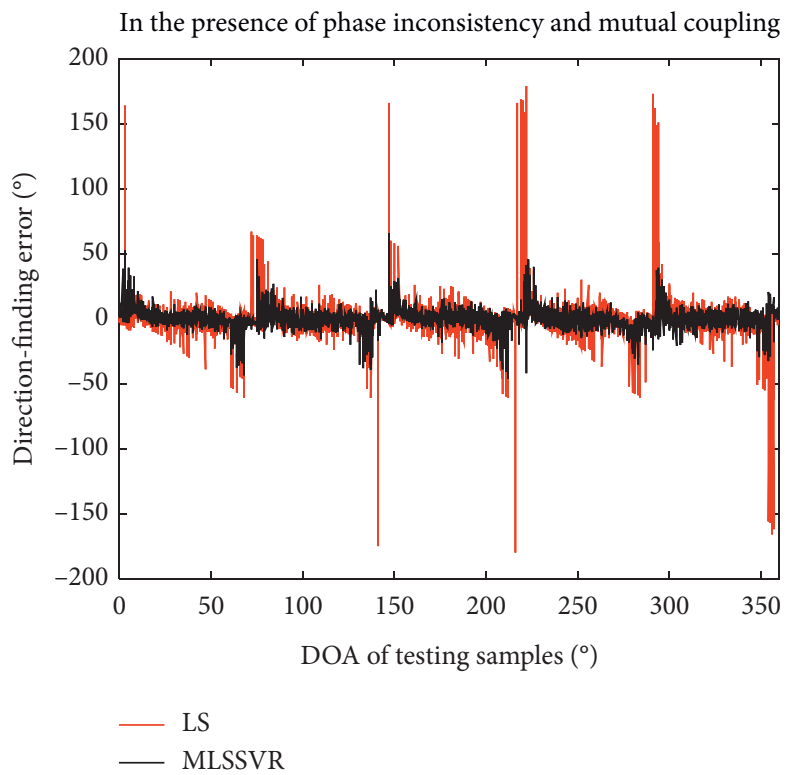

FIgURE 3: Direction-finding error of testing samples with phase inconsistency and mutual coupling.

mutual coupling are still in existence. For the same training data sets and testing samples, the two-layer convolutional neural network is used to estimate the DOA. The MaxEpochs is set to 15 (For the neural network model, it was obvious that the value of MaxEpochs in neural network could be larger to reduce the directionfinding error of the model. Here, a small value was deliberately selected to reduce the training time of neural network to approximate the training time of MLSSVR model.), so that the training time of the neural network model is close to the MLSSVR model. Figure 5 shows the direction-finding error of testing samples.

Table 3 shows the MLSSVR model is suitable for small training data sets, and compared with neural network model, the MLSSVR model ensures direction-finding accuracy while shortening training time. 


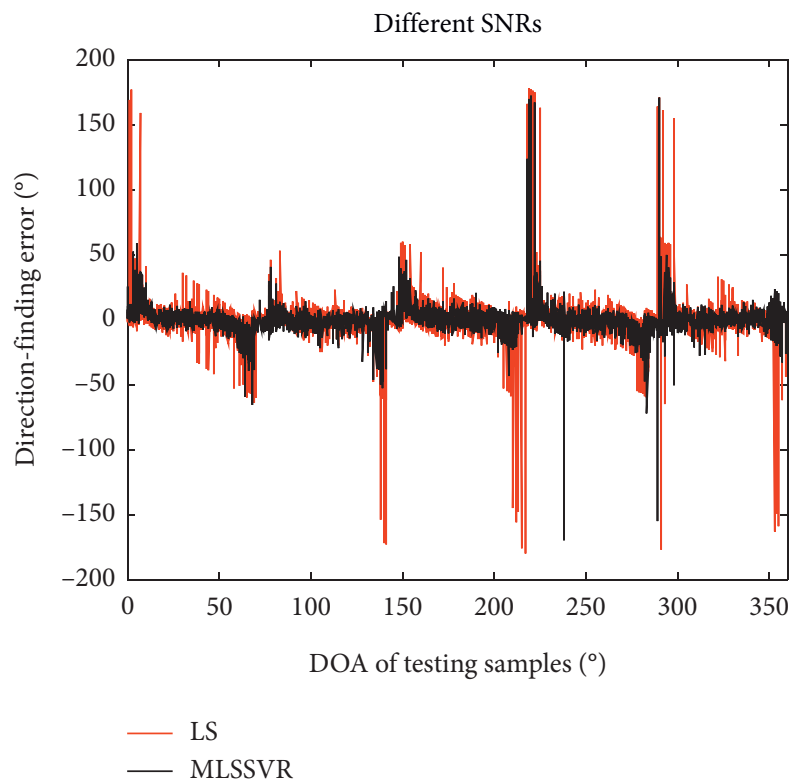

FIGURE 4: Direction-finding error of testing samples with different SNRs.

TABLE 2: RMSE of DOA of the testing samples.

\begin{tabular}{|c|c|c|c|c|c|}
\hline & The number of testing samples & Phase inconsistency & Mutual coupling & $\begin{array}{l}\text { Phase inconsistency mutual coupling } \\
\left.\text { RMSE of DOA ( }{ }^{\circ}\right)\end{array}$ & Different SNRs \\
\hline $\begin{array}{l}\text { LS } \\
\text { MLSSVR }\end{array}$ & 3600 & $\begin{array}{l}4.0870 \\
2.9695\end{array}$ & $\begin{array}{c}10.2529 \\
5.2498\end{array}$ & $\begin{array}{c}15.9103 \\
7.9172\end{array}$ & $\begin{array}{l}20.1085 \\
11.3850\end{array}$ \\
\hline
\end{tabular}

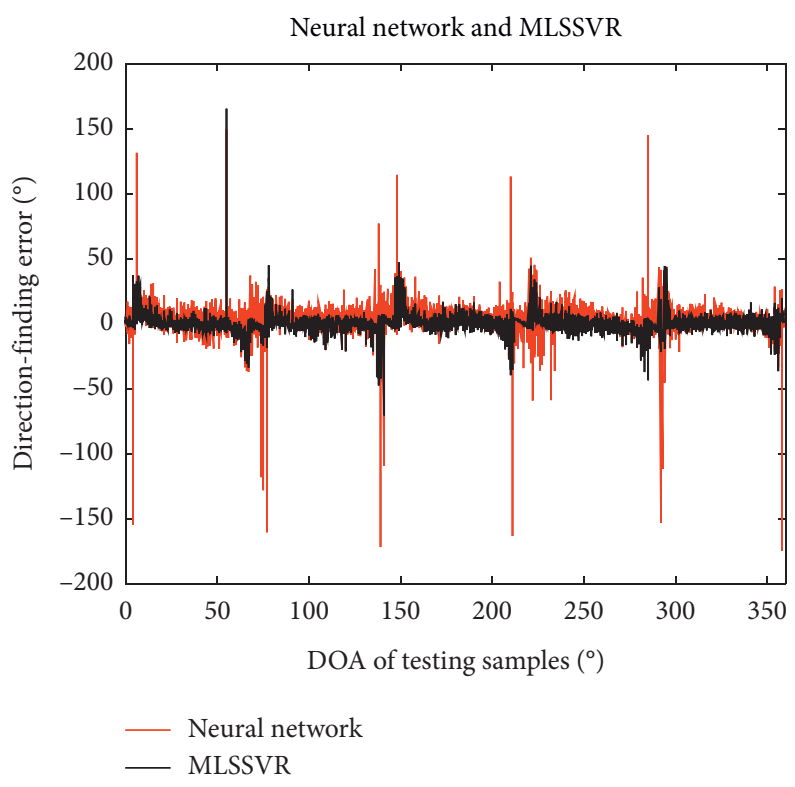

FIGURE 5: Direction-finding error of testing samples.

TABLE 3: RMSE of DOA of the testing samples.

\begin{tabular}{lcc}
\hline & RMSE of DOA $\left(^{\circ}\right)$ & Training times $(\mathrm{s})$ \\
\hline Neural network & 14.7228 & 2.531350 \\
MLSSVR & 7.7232 & 2.548054 \\
\hline
\end{tabular}




\section{Conclusion}

The standard formulation of support vector regression can only deal with the single-output case, and when it is applied to radio direction-finding, there may be a problem that the direction-finding results have large errors around $0^{\circ}$ or $360^{\circ}$. This paper applies the MLSSVR model to the field of radio direction-finding; the training data sets consist of phase differences of each baseline and the cosine and sine functions of each angle from $0^{\circ}$ to $360^{\circ}$. The DOA is calculated by the sine function and cosine function of the incident angle, thus avoiding a larger case finding the error results in the vicinity of $0^{\circ}$ or $360^{\circ}$. In the case of comprehensive disturbances in the direction-finding system, the effectiveness of the MLSSVR model is verified by numerical simulation. And with small training data sets, it can still effectively improve the direction-finding accuracy compared to the LS method.

\section{Data Availability}

All training data sets and testing samples used to support the findings of this study are available from the corresponding author upon request.

\section{Conflicts of Interest}

The authors declare that they have no conflicts of interest.

\section{Acknowledgments}

This work was supported by the No. 36 Research Institute of CETC under the project no. CX05.

\section{References}

[1] L. Wan, L. Sun, X. Kong, Y. Yuan, K. Sun, and F. Xia, “Taskdriven resource assignment in mobile edge computing exploiting evolutionary computation," IEEE Wireless Communications, vol. 26, no. 6, pp. 94-101, 2019.

[2] F. Wen, Z. Zhang, K. Wang, G. Sheng, and G. Zhang, "Angle estimation and mutual coupling self-calibration for ULAbased bistatic MIMO radar," Signal Processing, vol. 144, pp. 61-67, 2018.

[3] F. Wen and J. Shi, "Fast direction finding for bistatic EMVSMIMO radar without pairing," Signal Process, vol. 173, 2020.

[4] X. Wang, L. Wang, X. Li, and G. Bi, "Nuclear norm minimization framework for DOA estimation in MIMO radar," Signal Processing, vol. 135, pp. 147-152, 2017.

[5] L. Wan, X. Kong, and F. Xia, "Joint range-Doppler-angle estimation for intelligent tracking of moving aerial targets," IEEE Internet of Things Journal, vol. 5, no. 3, pp. 1625-1636, 2018.

[6] K. C. Ho and Y. T. Chan, "An asymptotically unbiased estimator for bearings-only and Doppler-bearing target motion analysis," IEEE Transactions on Signal Processing, vol. 54, no. 3, pp. 809-822, 2006.

[7] J.-H. Lee, J.-H. Lee, and J.-M. Woo, "Method for obtaining three- and four-element array spacing for interferometer direction-finding system," IEEE Antennas and Wireless Propagation Letters, vol. 15, pp. 897-900, 2016.

[8] J. H. Lee, J. K. Kim, H. K. Ryu, and Y. J. Park, "Multiple array spacings for an interferometer direction finder with high direction-finding accuracy in a wide range of frequencies," IEEE Antennas and Wireless Propagation Letters, vol. 17, no. 4, pp. 563-566, 2018.

[9] D. Meng, X. Wang, M. Huang, L. Wan, and B. Zhang, "Robust weighted subspace fitting for DOA estimation via block sparse recovery," IEEE Communications Letters, vol. 24, no. 3, pp. 563-567, 2020.

[10] J. Zheng, T. Yang, H. Liu, and T. Su, "Efficient data transmission strategy for IIoTs with arbitrary geometrical array," IEEE Transactions on Industrial Informatics, vol. 99, Article ID 2993586, 2020.

[11] J. Zheng, T. Yang, H. Liu, T. Su, and L. Wan, "Accurate detection and localization of UAV swarms-enabled MEC system," IEEE Transactions on Industrial Informatics, vol. 99, Article ID 3015730, 2020.

[12] C. S. Park and D. Y. Kim, “The fast correlative interferometer direction finder using I/Q demodulator,", in Proceedings of the Asia-Pacific Conference on Information Processing, pp. 1-5, Busan, South Korea, 2006.

[13] G. Zhu, Y. Wang, and S. Mi, "Research on direction finding technique based on the combination of weighted least squares method and MUSIC algorithm," Rader Science and Technology, vol. 17, no. 3, pp. 319-323, 2019.

[14] J. Zheng, H. Liu, and Q. H. Liu, "Parameterized centroid frequency-chirp rate distribution for LFM signal analysis and mechanisms of constant delay introduction," IEEE Transactions on Signal Processing, vol. 65, no. 24, pp. 6435-6447, 2017.

[15] B. Friedlander and A. J. Weiss, "Direction finding in the presence of mutual coupling," IEEE Transactions on Antennas and Propagation, vol. 39, no. 3, pp. 273-284, 1991.

[16] T. Svantesson, "Modeling and estimation of mutual coupling in a uniform linear array of dipole," in Proceedings of the IEEE International Conference on Acoustics, Speech, and Signal Processing, IEEE, Phoenix, AZ, USA, pp. 2961-2964, March 1999.

[17] M. Lin and L. Yang, "Blind calibration and DOA estimation with uniform circular arrays in the presence of mutual coupling," IEEE Antennas and Wireless Propagation Letters, vol. 5, no. 1, pp. 315-318, 2006.

[18] A. J. Weiss and B. Friedlander, "Array shape calibration using eigen-structure methods," Signal Processing, vol. 22, no. 3, pp. 251-258, 1991.

[19] B. P. Flanagan and K. L. Bell, "Array self-calibration with large sensor position errors," Signal Processing, vol. 81, no. 10, pp. 2201-2214, 2001.

[20] A. Paulraj and T. Kailath, "Direction of arrival estimation by eigen-structure methods with unknown sensor gain and phase," Acoustics, Speech, and Signal Processing," in Proceedings of the ICASSP'85. IEEE International Conference on Acoustics, Speech, and Signal Processing, pp. 640-643, IEEE, Tampa, FL, USA, April 1985.

[21] Y. Li and M. Er, "Theoretical analyses of gain and phase error calibration with optimal implementation for linear equispaced array," IEEE Transactions on Signal Processing, vol. 54, no. 2, pp. 712-723, 2006.

[22] Q. Zhai, "Research on least square direction-finding method," Radio Engineering, vol. 38, no. 3, pp. 55-57, 2008.

[23] C. S. Shieh and C. T. Lin, "Direction of arrival estimation based on phase differences using neural fuzzy network," IEEE Transactions on Antennas and Propagation, vol. 48, no. 7, pp. 1115-1124, 2000.

[24] C. G. Christodoulou, J. A. Rohwer, and C. T. Abdallah, "The use of machine learning in smart antennas," in Proceedings of 
the IEEE Antennas and Propagation Society Symposium, pp. 321-324, IEEE, Monterey, CA, USA, June 2004.

[25] J. A. Rohwer, C. T. Abdallah, and C. G. Christodoulou, "Least squares support vector machines for direction of arrival estimation with error control and validation," in Proceedings of the GLOBECOM'03. IEEE Global Telecommunications Conference, pp. 2172-2176, IEEE, San Francisco, CA, USA, December 2003.

[26] C. A. M. Lima, C. Junqueira, R. Suyama, F. J. V. Zuben, and J. M. T. Romano, "Least-square support vector machines for DOA estimation: a step-by-step description and sensitivity analysis," in Proceedings of the International Joint Conference on Neural Networks, pp. 3226-3231, Montreal, Canada, July 2005.

[27] S. Vigneshwaran, N. Sundararajan, and P. Saratchandran, "Direction of arrival (DOA) estimation under array sensor failures using a minimal resource allocation neural network," IEEE Transactions on Antennas and Propagation, vol. 55, no. 2, pp. 334-343, 2007.

[28] M. Dehghanpour, V. T. T. Vakili, and A. Farrokhi, "DOA estimation using multiple kernel learning SVM considering mutual coupling," in Proceedings of the 2012 Fourth International Conference on Intelligent Networking and Collaborative Systems, pp. 55-61, Bucharest, Romania, September 2012.

[29] R. Wang, B. Wen, and W. Huang, "A support vector regression-based method for target direction of arrival estimation from HF radar data," IEEE Geoscience and Remote Sensing Letters, vol. 15, no. 5, pp. 674-678, 2018.

[30] Z.-M. Liu, C. Zhang, and P. S. Yu, "Direction-of-arrival estimation based on deep neural networks with robustness to array imperfections," IEEE Transactions on Antennas and Propagation, vol. 66, no. 12, pp. 7315-7327, 2018.

[31] S. Abeywickrama, L. Jayasinghe, H. Fu, S. Nissanka, and C. Yuen, "RF-based direction finding of UAVs uing DNN," in Proceedings of the IEEE International Conference on Communication Systems (ICCS), December 2018.

[32] S. Xu, X. An, X. Qiao, L. Zhu, and L. Li, "Multi-output leastsquares support vector regression machines," Pattern Recognition Letters, vol. 34, no. 9, pp. 1078-1084, 2013. 